\title{
Intruder Dynamics on Vibrofluidized Granular Surfaces
}

Alexander D. Wissner-Gross ${ }^{1,2}$

${ }^{1}$ Department of Physics, Harvard University, Cambridge, MA 02138-2933, U.S.A.

${ }^{2}$ Center for the Environment, Harvard University, Cambridge, MA 02138-2902, U.S.A.

\section{ABSTRACT}

Granular fluids have received significant attention for their transport characteristics within rigid boundaries. In this work, we study the potential for amorphous granular beds themselves to serve as elastic boundaries for transport and manipulation of surface particles. In particular, we measure the effect of the mass and diameter of an intruding particle, supported and tossed by a periodic vibrofluidized granular bed, on its horizontal self-diffusivity using a twodimensional hard-sphere molecular dynamics simulation. Intruder mass and diameter scaling exponents are fitted and found to be consistent with dominant Stokes drift. Intruder types favorable for manipulation applications are identified.

\section{INTRODUCTION}

Granular fluids are an industrially and scientifically relevant class of vibrationally programmable matter [1]. Thin layers of hard spheres in a vertically oscillated container have demonstrated a rich set of spatial patterns beyond Faraday wave predictions that can be selected by tuning the container floor's oscillation frequency and amplitude [2-4]. More complex patterns can be selected with multiple frequency forcing [4]. In the continuum limit, arbitrary wave fields have even been demonstrated in traditional liquid tanks [5].

Such programmability suggests that granular fluid patterns might be harnessed as force fields for parallel manipulation tasks. Parallel manipulation of small numbers of parts using many degrees of actuation has received considerable theoretical [6-7] and experimental [8] attention. Parallel positional control of free bodies as short as $3 \mathrm{~mm}$ and as light as $8.6 \mathrm{~g}$ has been achieved using arrays of MEMS actuators [9]. However, the inverse problem of manipulating large numbers of parts with only a few degrees of actuation ("minimalist manipulation") has received less attention. The most noteworthy accomplishment in this direction has been the demonstration of a planar manipulator with global rotational and translational degrees of freedom [10]. So far, such planar manipulators have only been able to positionally address centimeter-scale objects, necessitating new methods for minimalist manipulation at smaller length scales. Controlled tossing of surface impurities by patterned granular fluids is one promising approach, since granule diameters can be scaled down to $100 \mu \mathrm{m}$ while preserving pattern formation [11].

While the vertical dynamics of a large intruder particle submerged in a deep granular bed have been investigated previously (e.g., in connection with the Brazil nut effect), both experimentally $[12,13]$ and numerically [14], the dynamics of an intruder at or near the surface of a thin granular layer have not been addressed. This report addresses the dynamics of surface impurities on vibrofluidized granular patterns with two-dimensional numerical simulations.

\section{THEORY}


Consider, as a model for surface intruder dynamics, self-diffusion in a standing wave potential

$$
U(x, t)=A \cos (2 \pi x / \lambda) \sin (\pi f t)=\frac{-A}{2}\left[\sin \left(k_{1} x-\omega_{1} t\right)+\sin \left(k_{2} x-\omega_{2} t\right)\right]
$$

for pattern wavelength $\lambda$, container vibration frequency $f, \omega_{1}=\omega_{2}=\pi f$, and $k_{1}=-k_{2}=2 \pi / \lambda$, when parameterized by horizontal self-diffusion coefficient $D$, defined by

$$
D \equiv \frac{1}{2} \lim _{t \rightarrow \infty} \frac{\left\langle\left[x\left(t_{0}+t\right)-x\left(t_{0}\right)\right]^{2}\right\rangle}{t}
$$

Since $U(x, t)$ is the sum of equal counter-propagating waves, no intruder drift velocity (Stokes drift) can be induced without diffusion [15].

However, discrete collisions of a large surface intruder with supporting granules constitute a diffusion mechanism. The drift velocity including diffusion [15] is given by

$$
v \propto\left(1+\alpha_{1}^{2}\right)^{-1}-\left(1+\alpha_{2}^{2}\right)^{-1}
$$

where $\alpha_{i}=D\left|k_{i}\right|^{2} \omega_{i}^{-1}$ and $i=1,2$. For a pure standing wave, this expression vanishes. Consider therefore an imperfect standing wave with $k_{1}+k_{2}=\delta k$, which is more realistic for granular patterns. In this case, it follows that

$$
v \propto \frac{D^{2} \omega_{1}^{-2}\left|k_{1}\right|^{3} \delta k}{\left(1+\left(D \omega_{1}^{-1} k_{1}^{2}\right)^{2}\right)^{2}} .
$$

If diffusion dominates the classical Stokes drift, then $\alpha_{1}>>1$ and $v \propto D^{-2}$, while if the classical drift dominates, then $\alpha_{1}<<1$ and $v \propto D^{2}$. In the case of surface intruder tossing, classical drift is expected to dominate, since the intruder will spend much of its time in free flight above the granular bed. The acceleration of an intruder in contact with a rising pile of granules will be proportional to its diameter and inversely proportional to its mass (assuming constant bed pressure), so $v \propto m_{I}^{-1} d$ and $D \propto m_{I}^{-0.5} d_{I}^{0.5}$.

\section{SIMULATION DETAILS}

A hard-disk two-dimensional molecular dynamics simulation was constructed, incorporating angular momentum, friction, and inelastic collapse avoidance [16]. The simulated container had a high ceiling and width $W$ delimited by fixed horizontal boundaries. At the beginning of each trial run, a granular bed of $N$ monodisperse disks with diameter $d_{0}$ was initialized. Granules were initially placed in a square lattice with separation $2 d_{0}$, horizontal and vertical velocity components randomly distributed in the interval $[-0.1,0.1] \mathrm{ms}^{-1}$, and no angular momentum. 
The simulation was event-driven. A calendar with the next scheduled collision event for each granule was kept, and selectively recalculated after each collision [17]. In collisions of granules with container walls and each other, a velocity-dependent coefficient of restitution [18],

$$
\varepsilon(v)=1-\varepsilon_{0}\left(v / v_{0}\right)^{0.2}
$$

was used where $v$ is the component of the relative velocity normal to the tangent plane of collision, $v_{0}=1.0 \mathrm{~ms}^{-1}$. Additional energy was dissipated by friction, parameterized by the coefficient $\mu$. Following Rapaport [19], relative tangential restitution above a critical impact angle was eliminated, with a maximum coefficient of tangential restitution, $\beta_{0}$. Inelastic collapse was avoided by setting $\varepsilon(v)=1.0$ below a cutoff $v_{\text {crit }}=10^{-6} \mathrm{~ms}^{-1}$. The collision parameters used for these experiments are summarized in Table I.

Table I. Parameters used for inelastic collisions.

\section{Parameter Granule-granule Granule-boundary}

$\begin{array}{lll}\mu & 0.2 & 0.2 \\ \beta_{0} & 0.0 & 0.0 \\ \varepsilon_{0} & 0.4 & 0.2\end{array}$

By convention, the floor height $y(t)$ of a vibrated container evolves as

$$
y(t)=-\Gamma g(2 \pi f)^{-2} \cos (2 \pi f t)
$$

for frequency $f$ and dimensionless amplitude $\Gamma$. To determine the accuracy of the numerical implementation, the dependence of $\lambda$ on $f, \Gamma$, and $N$ was measured. These tests were performed in an aperiodic container with $d_{0}=1.5 \mathrm{~mm}, W=100 d_{0}, N=600, \Gamma=3.6$, and $f=10 \mathrm{~Hz}$, unless otherwise varied. Wavelengths were extracted by calculating the first nonzero peak in the autocorrelation function of the granules' horizontal distribution:

$$
C_{x, x}\left(\lambda^{\prime}\right)=\left(W-\lambda^{\prime}\right)^{-1} \int_{0}^{W} h(x) h\left(x+\lambda^{\prime}\right) d x
$$

The horizontal distribution was found by integrating granule positions over $10 \mathrm{~s}$. Previous results [18] were well reproduced across a broad parameter range.

The main experiment was conducted with parameter values $\Gamma=3.6, f=10 \mathrm{~Hz}, g=9.8$ $\mathrm{ms}^{-2}, N=180$, and $d_{0}=1.5 \mathrm{~mm}$. The horizontally periodic container, with $W=30 d_{0}$, was chosen to favor the fundamental mode of the 6-layer bed oscillated at the given values of $f$ and $\Gamma$ [16], in which at most one pattern peak existed at any time. In addition to the computational benefit of this container geometry, the granular force field resembled the desired application of multiple intruders being consecutively tossed by a single peak.

The time evolutions of intruders were measured. The granular fluid was first given $t_{0}=$ $0.6 \mathrm{~s}$ to develop patterns, after which one diameter $d_{0}$ and mass $m_{0}$ granule was removed and reintroduced as an intruder with diameter $d_{I}$ and mass $m_{I}$. The intruder was created at rest at a height of $40 d_{0}$ above the mean floor position, and horizontally centered (an undistinguished position, as the boundary conditions were periodic). Simulations were halted after 2 s. For each 
combination of $d_{I} / d_{0}=1,3,5, \ldots, 23$ and $m_{I} / m_{0}=1,2,4,8,100$ trials were conducted in which the initial granule velocities were randomized. An example of an intruder being tossed is shown in Figure 1.

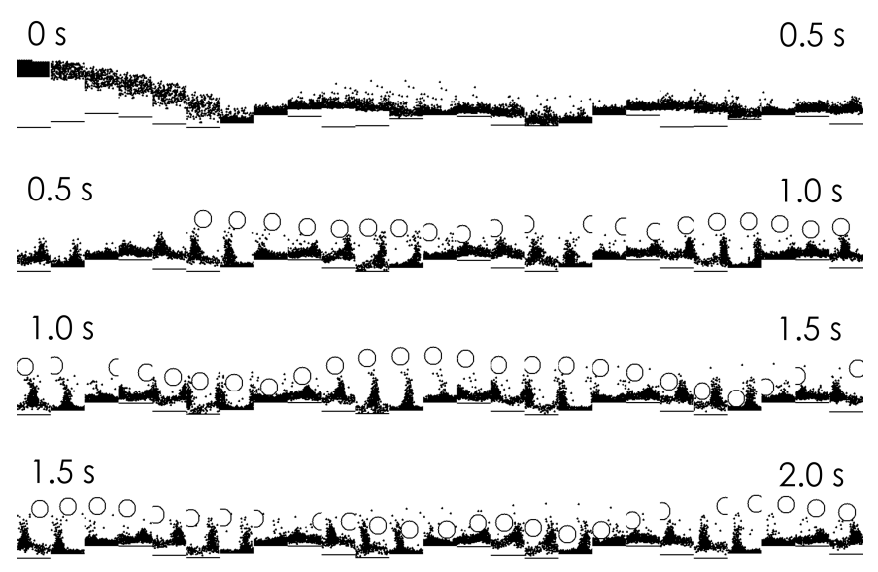

Figure 1. Time evolution of intruder $\left(m_{I} / m_{0}=1, d_{l} / d_{0}=15\right)$ tossed by a granular pattern over a 2-s interval. Note that intruder (large disk) was only introduced after supporting pattern formed.

\section{DISCUSSION}

The mean square horizontal displacement $\left\langle\Delta x^{2}\right\rangle$ of the intruder ensemble over these trials was calculated as a function of time over their 1.4-s existence. At $m_{I}=m_{0}$ and $d_{I} \geq 5 d_{0}$, the time evolution of $\left\langle\Delta x^{2}\right\rangle$ was approximately linear after $0.1 \mathrm{~s}$ and consistent with Brownian diffusion (see Figure 2). Self-diffusion coefficients were therefore fitted to the last $1.2 \mathrm{~s}$ of $\left\langle\Delta x^{2}\right\rangle$, ignoring the first $0.2 \mathrm{~s}$ of ensemble relaxation.

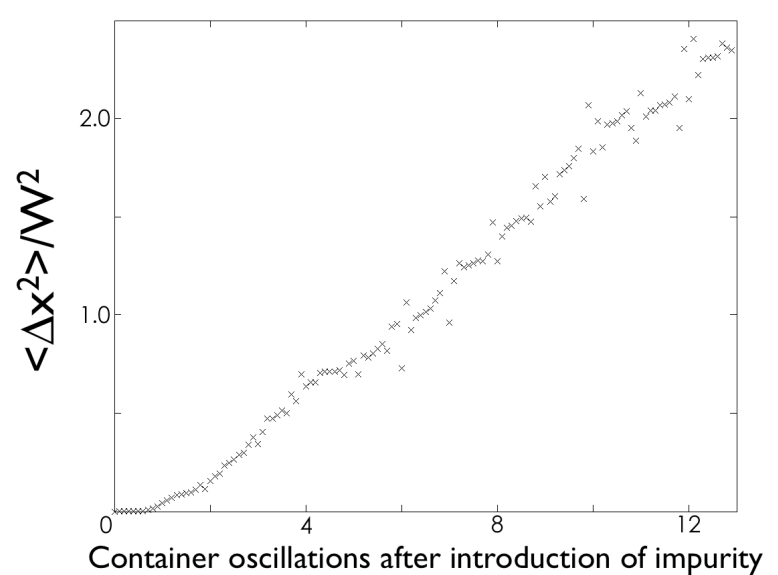

Figure 2. Example evolution of intruder ensemble's mean square horizontal displacement $\left(m_{I} / m_{0}\right.$ $\left.=1, d_{I} / d_{0}=7\right)$, normalized by the squared container width, $W$.

The self-diffusion coefficients calculated for each intruder diameter and mass are shown in Figure 3. The diffusivity relation was fit by the power law 


$$
D=k\left(m_{I} / m_{0}\right)^{\alpha}\left(d_{I} / d_{0}\right)^{\beta},
$$

for $k=(19.1 \pm 2.8) \mathrm{cm}^{2} \mathrm{~s}^{-1}, \alpha=-0.74 \pm 0.07$, and $\beta=0.37 \pm 0.06$. The fitted exponent $\beta$ was more consistent with the 0.5 exponent expected from dominant Stokes drift than the -0.5 exponent expected from dominant diffusion in a continuum. The fitted value of $\alpha$ was $3.5 \sigma$ away from the -0.5 value expected for intruder tossing.

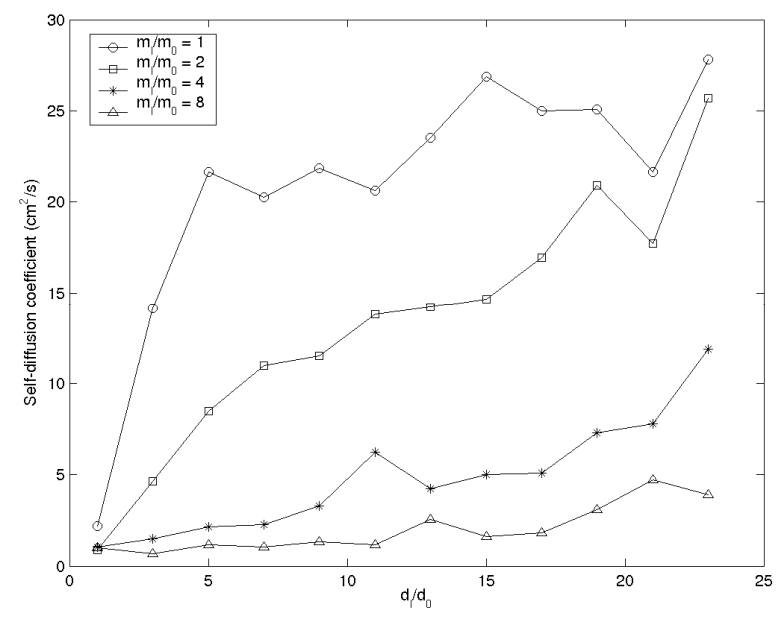

Figure 3. Horizontal self-diffusion rates for various relative intruder masses, $m_{I} / m_{0}$, and diameters, $d_{I} / d_{0}$.

For the intruder to remain near a single trough in the granular bed, it should remain within $\pm \lambda / 4$ of the trough's horizontal position, because the distance between a peak and the adjacent peak that rises after time $T$ is $\lambda / 2$. Hence, the desirable self-diffusivities are constrained:

$$
D \leq(\lambda / 2)^{2} /(2 T)=f \lambda / 8 .
$$

For the standard parameters [18] of the main experiment reported here, the intruder types that are positionally stable are constrained by

$$
\frac{f \lambda}{\left(m_{I} / m_{0}\right)^{\alpha}\left(d_{I} / d_{0}\right)^{\beta}} \geq 152.8 \mathrm{~cm}^{2} \mathrm{~s}^{-1} .
$$

Additionally, the average height of the bottom of the intruder was measured as a function of diameter for various masses. For $d_{I} \geq 5 d_{0}$, the bottom height saturated at $\sim 25 d_{0}$, indicating that the intruder was, in fact, supported by the granular bed and did not crush it.

\section{RESULTS AND CONCLUSIONS}

The horizontal self-diffusivities of surface impurities of various diameters and masses were found to scale with mass exponent $-0.74 \pm 0.07$ and diameter exponent $0.37 \pm 0.06$, 
consistent with dominant Stokes drift. A constraint on the relative masses and diameters of impurities that diffused slowly enough to remain horizontally stable over multiple container oscillations was derived.

This result is a step toward granular fluidic manipulation, in which the container oscillation function is tuned to individually address the motion of multiple impurities of different masses and diameters. In particular, by varying the container frequency, intruder particles might be made to cross this boundary in mass-diameter phase space and be immobilized, while a few are freed for manipulation by additional degrees of freedom. A better understanding of such degrees of freedom as horizontal shaking [20] should therefore be relevant for future studies.

\section{ACKNOWLEDGMENTS}

The author thanks the Fannie and John Hertz Foundation and the Harvard University Center for the Environment for research funding, and J. C. Ellenbogen for helpful discussion.

\section{REFERENCES}

1. G. H. Ristow, Pattern Formation in Granular Materials (Springer-Verlag, New York, 2000) pp. 1-10.

2. F. Melo, P. Umbanhowar, and H. L. Swinney, Phys. Rev. Lett. 72, 172-175 (1994).

3. J. M. Schleier-Smith and H. A. Stone, Phys. Rev. Lett. 86, 3016-3019 (2001).

4. H. S. Wi, K. Kim, and H. K. Pak, J. Korean Phys. Soc. 38, 573-576 (2001).

5. S. Naito, M. Minoura, M. Takeda, and E. Okuyama, Int. J. Offshore Polar 16, 10-17 (2006).

6. J. E. Luntz, W. Messner, and H. Choset in Distributed Manipulation, edited by K. F. Böhringer and H. Choset (Kluwer Academic Publishers Group, Norwell, MA, 2000), pp. 103-126.

7. K. F. Böhringer, B. R. Donald, L.E. Kavraki, and F. Lamiraux, IEEE Trans. Robot. Auto. 16, 157-170 (1999).

8. M. Yim and A. Berlin in ICRA Workshop on Distributed Manipulation (1999).

9. J. W. Suh, R. B. Darling, K.-F. Böhringer, B. R. Donald, H. Baltes, and G. T. A. Kovacs, Proc. IEEE Int. Conf. Robot. Auto. 2, 1101-1108 (2000).

10. D. S. Reznik, PhD. Thesis, University of California at Berkeley, 2000.

11. M. V. Sapozhnikov, Y. V. Tolmachev, I. S. Aranson, and W.-K. Kwok. Phys. Rev. Lett. 90, $114301(2003)$.

12. T. Shinbrot and F. J. Muzzio, Phys. Rev. Lett. 81, 4365-4368 (1998).

13. J. B. Knight, H. M. Jaeger, and S. R. Nagel, Phys. Rev. Lett. 70, 3728-3731 (1993).

14. R. Jullien and P. Meakin, Phys. Rev. Lett. 69, 640-643 (1992).

15. K. M. Jansons and G. D. Lythe, Phys. Rev. Lett. 81, 3136-3139 (1998).

16. E. Clément and L. Labous, Phys. Rev. E 62, 8314-8323 (2000).

17. H. Gould and J. Tobochik, An Introduction to Computer Simulation Methods: Applications to Physical Systems, 2nd ed. (Benjamin Cummings, San Francisco, 1996), pp. 272-318.

18. S. Luding, E. Clément, J. Rajchenbach, and J. Duran, Europhys. Lett. 36, 247-252 (1996).

19. D. C. Rapaport, The Art of Molecular Dynamics Simulations (Cambridge University Press, New York, 1995), pp. 307-314.

20. G. Metcalfe, S. G. K. Tennakoon, L. Kondic, D. G. Schaeffer, and R. P. Behringer, Phys. Rev. E 65, 031302 (2002). 